\title{
Pattern of Extraocular Muscle Involvements in Miller Fisher Syndrome
}

\author{
Won Yeol Ryu ${ }^{a}$ \\ Yoo Hwan Kim ${ }^{\mathrm{b}}$ \\ Byeol-A Yoon ${ }^{\mathrm{c}, \mathrm{d}}$ \\ Hwan Tae Park ${ }^{\mathrm{d}, \mathrm{e}}$ \\ Jong Seok Bae ${ }^{* *}$ \\ Jong Kuk Kim ${ }^{\mathrm{cd} *}$ \\ aDepartments of Ophthalmology, \\ 'Neurology, and ${ }^{\mathrm{e} M o l e c u l a r}$ Neuroscience, \\ Dong-A University College of Medicine, \\ Busan, Korea \\ bDepartment of Neurology, \\ Hangang Sacred Heart Hospital, \\ Hallym University College of Medicine, \\ Seoul, Korea \\ dPeripheral Neuropathy Research Center, \\ Dong-A University College of Medicine, \\ Busan, Korea \\ fDepartment of Neurology, \\ Kangdong Sacred Heart Hospital, \\ Hallym University College of Medicine, \\ Seoul, Korea
}

\begin{abstract}
Background and Purpose The most-common initial manifestation of Miller Fisher syndrome (MFS) is diplopia due to acute ophthalmoplegia. However, few studies have focused on ocular motility findings in MFS. This study aimed to determine the pattern of extraocular muscle (EOM) paresis in MFS patients.

Methods We consecutively recruited MFS patients who presented with ophthalmoplegia between 2010 and 2015. The involved EOMs and the strabismus pattern in the primary position were analyzed. Antecedent infections, other involved cranial nerves, and laboratory findings were also reviewed. We compared the characteristics of the patients according to the severity of ophthalmoplegia between complete ophthalmoplegia (CO) and incomplete ophthalmoplegia $(\mathrm{IO})$.

Results Twenty-five patients ( 15 males and 10 females) with bilateral ophthalmoplegia were included in the study. The most-involved and last-to-recover EOM was the lateral rectus mus-

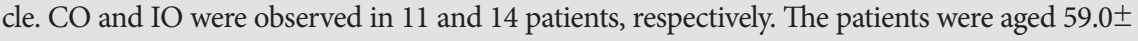
18.4 years (mean $\pm \mathrm{SD})$ in the $\mathrm{CO}$ group and $24.9 \pm 7.4$ years in the IO group $(p<0.01)$, and comprised $63.6 \%$ and $21.4 \%$ females, respectively $(p=0.049$ ). Elevated cerebrospinal fluid protein was identified in $60.0 \%$ of patients with CO and $7.7 \%$ of patients with IO $(p=0.019)$ for a mean follow-up time from the initial symptom onset of 3.7 days.

Conclusions The lateral rectus muscle is the most-involved and last-to-recover EOM in ophthalmoplegia. The $\mathrm{CO}$ patients were much older and were more likely to be female and have an elevation of cerebrospinal fluid protein than the IO patients.

Key Words ophthalmoplegia, miller fisher syndrome, guillain-barré syndrome, GQ1b ganglioside.
\end{abstract}

$\begin{array}{ll}\text { Received } & \text { May 17, 2018 } \\ \text { Revised } & \text { December 27, 2018 } \\ \text { Accepted } & \text { December 27, 2018 }\end{array}$

\section{Correspondence}

Jong Seok Bae, MD, $\mathrm{PhD}$

Department of Neurology,

Kangdong Sacred Heart Hospital,

Hallym University College of Medicine,

150 Seongan-ro, Gangdong-gu,

Seoul 05355, Korea

Tel +82-2-478-6330

Fax +82-2-2224-2854

E-mail jsb_res@hotmail.co.kr

Jong Kuk Kim, MD, PhD

Department of Neurology,

Peripheral Neuropathy Research Center,

Dong-A University College of Medicine,

26 Daesingongwon-ro, Seo-gu,

Busan 49201, Korea

Tel +82-51-244-8338

Fax +82-51-240-5266

E-mail advania9@chol.com

*These authors contributed equally to this work.

\section{INTRODUCTION}

Miller Fisher syndrome (MFS) is a representative regional variant of Guillain-Barré syndrome (GBS) that is characterized by the clinical triad of ophthalmoplegia, ataxia, and areflexia. ${ }^{1}$ However, this characteristic triad is not present in all MFS patients, with many patients exhibiting only two signs or only ophthalmoplegia. Among the triad, the mostcommon initial symptom is ophthalmoplegia. MFS has been previously shown to cause bilateral symmetric ophthalmoplegia. ${ }^{1,2}$ Recent studies have found limitation of abduction in both eyes to be a typical form of ophthalmoplegia in MFS, with complete ophthalmoplegia (CO) observed in only one-third of patients. ${ }^{3,4}$

Many clinical and immunologic studies have investigated MFS since the discovery of the anti-GQ1b-ganglioside antibody. ${ }^{1,4-10}$ However, few studies-and especially ones conducted by neuro-ophthalmologists-have systematically explored ocular motility, even though ophthalmoplegia is the most-common, disabling, and consistent finding in MFS. Two previous

\footnotetext{
(c) This is an Open Access article distributed under the terms of the Creative Commons Attribution Non-Commercial License (https://creativecommons.org/licenses/by-nc/4.0) which permits unrestricted non-commercial use, distribution, and reproduction in any medium, provided the original work is properly cited.
} 
representative studies investigating acute ophthalmoplegia without ataxia with positivity for anti-GQ1b-ganglioside antibody revealed the pattern of oculomotor paresis in a subset of MFS patients. ${ }^{2,3}$ However, there have been few analyses of how the clinical findings vary with the severity of ophthalmoplegia among all MFS patients. The aim of the present study was to delineate the clinical characteristics of extraocular muscle (EOM) paresis and correlate these characteristics with other accompanying symptoms and signs in MFS patients.

\section{METHODS}

We consecutively enrolled patients who were diagnosed with MFS or an associated disease and treated between 2010 and 2015 at our medical center. In 2010, a neuro-ophthalmologist (W.Y.R.), who is a strabismus surgery specialist, joined the neuroimmunology team and began performing systematic observations of ocular motility findings in MFS patients. This study was approved by the Institutional Review Board of Dong-A University Hospital (approval no. DAUHIRB-16-083). Informed consent was obtained from each participant, and all participants were treated in accordance with the tenets of the Declaration of Helsinki.

Because there is no current universal consensus regarding the diagnosis of MFS, we referred to the recent diagnostic classification of the GBS Classification Group. ${ }^{11}$ We subsequently established the following inclusion criteria based on the ocular motility findings of MFS patients being the main focus in this study: 1) clinically typical MFS with the classic triad regardless of positivity for immunoglobulin $\mathrm{G}$ (IgG) anti-GQ1b-ganglioside antibody and 2) acute ophthalmoplegia with either ataxia or areflexia/hyporeflexia and positivity for IgG anti-GQ1b-ganglioside antibody. The symptoms and signs were acute and progressive within 4 weeks in all cases. To reduce confusion due to an overlapping syndrome, we excluded ophthalmoplegic GBS with prominent limb weakness (severity of at least Medical Research Council grade 4). Patients with Bickerstaff's brainstem encephalitis were also excluded from this study because it is very rare (there was only one case in our series during the study period), and it usually makes the interpretation of ocular motility findings difficult due to the presence of altered mentality.

To determine the clinical characteristics of ophthalmoplegia in the MFS patients, we investigated the pattern of ocular deviation in the primary position, involved EOMs, presence of internal ophthalmoplegia, duration from the beginning of diplopia to the initial recovery of extraocular movement, whether the patient was treated with intravenous immunoglobulin (IVIg), number of days from the beginning of the IVIg treatment to the initial recovery of extraocular movement, and the final EOM that remained as an ocular limitation. In addition, we compared the patients according to the severity of ophthalmoplegia between $\mathrm{CO}$ and incomplete ophthalmoplegia (IO), including analyzing differences in various factors between these two groups. We defined CO as the combination of bilateral ptosis and paralysis of all directional extraocular movement regardless of internal ophthalmoplegia; the remaining cases not fulfilling these CO criteria were assigned to the IO group. ${ }^{12,13}$ Because it is difficult to clearly distinguish the involvement of the oblique muscles, these muscles were not analyzed in this study.

We also reviewed the following clinical and laboratory data: age, sex, seasonal distribution of occurrence, antecedent infection, interval from infection to initial symptom, other involved cranial nerves, cerebrospinal fluid (CSF) analysis, and antiganglioside antibody status. Albuminocytologic dissociation was defined as a high level of protein without an abnormal cell elevation in the CSF. The reference values used for the cell counts and protein levels of CSF were $<10 / \mu \mathrm{L}$ and $45 \mathrm{mg} / \mathrm{dL}$, respectively.

An antiganglioside antibody assay was applied to acutestage serum obtained within 2 weeks of symptom onset. An enzyme-linked immunosorbent assay (ELISA) was used to detect the anti-GQ1b-ganglioside antibody, as described previously with some modifications. ${ }^{14}$ The presence of the antiGQ1b-ganglioside antibody was determined by researchers (B.A.Y. and H.T.P.) at the Dong-A University Neuroimmunology Team who were blinded to the clinical information of the patients.

All statistical analyses were conducted using SPSS software (SPSS for Windows, version $12.0 \mathrm{~K}$, SPSS Inc., Chicago, IL, USA). Mann-Whitney U tests and Fisher's exact tests were used to compare the clinical findings between the $\mathrm{CO}$ and IO groups. Probability values of $p<0.05$ were considered to indicate statistical significance.

\section{RESULTS}

This study included 25 patients (15 males and 10 females). Twelve (48.0\%) patients developed MFS during the spring, mostly in May. Twenty (80.0\%) patients exhibited typical MFS with the classic triad, and five (20\%) patients exhibited a partial form as an anti-GQ1b antibody syndrome, such as ophthalmoplegia with either ataxia or areflexia/hyporeflexia (Table 1). Four of the patients were previously reported on as a case series. ${ }^{7}$

All subjects in the present study had bilateral ophthalmoplegia involving the lateral rectus muscle (LR) (25 patients, $100 \%$ ), followed by the superior (15 patients, $60.0 \%$ ), inferior (13 patients, $52.0 \%$ ), and medial (12 patients, $48.0 \%$ ) 
rectus muscles, with similar involvement frequencies. Regarding the degree of paralysis of the EOM, 14 (56.0\%) patients showed IC and only 11 (44.0\%) patients exhibited $\mathrm{CO}$. The most-frequent form of ocular deviation was esotropia (16 patients, 64.0\%). Exotropia was observed in seven (28.0\%) patients and vertical strabismus was found in only

Table 1. Characteristics of the patients included in this study

\begin{tabular}{|c|c|}
\hline Characteristics & $n(\%)$ \\
\hline Total patients & 25 \\
\hline Sex, male:females & 15 (60.0):10 (40.0) \\
\hline Age, years & $39.5 \pm 21.2$ (15 to 75$)$ \\
\hline Antecedent infection & $22(88.0)$ \\
\hline Upper respiratory symptoms & $12(54.5)$ \\
\hline Gastrointestinal symptoms & $10(45.5)$ \\
\hline Time from infection to initial symptom, days & $8.7 \pm 3.4$ (2 to 17$)$ \\
\hline $\begin{array}{l}\text { Classic MFS: partial form with anti-GQ1b } \\
\text { antibody syndrome }\end{array}$ & $20(80.0): 5(20.0)$ \\
\hline \multicolumn{2}{|l|}{ Laboratory tests } \\
\hline Positivity for lgG anti-G01b antibody & 18/24 (75.0) \\
\hline CSF albuminocytologic dissociation & $7 / 23(30.4)^{*}$ \\
\hline CSF protein, $\mathrm{mg} / \mathrm{dL}$ & $43.8 \pm 18.0^{*}$ \\
\hline Time from onset to CSF analysis, days & $3.7 \pm 2.1(1 \text { to } 9)^{*}$ \\
\hline IVlg-treated patients & $21 / 25(84.0)$ \\
\hline $\begin{array}{l}\text { Time to complete recovery from initial } \\
\text { symptom onset, weeks }\end{array}$ & $13.2 \pm 7.6$ (4 to 28$)$ \\
\hline
\end{tabular}

Data are $n, n(\%)$, mean \pm SD, or mean \pm SD (range) values.

*The CSF analysis included 23 of the 25 patients since 2 patients did not consent to the test

IgG: immunoglobulin G, IVlg: intravenous immunoglobulin, MFS: Miller Fisher syndrome. two (8.0\%) patients. Additionally, 10 (40.0\%) patients exhibited internal ophthalmoplegia and $10(40.0 \%)$ patients exhibited blepharoptosis. None of the included patients presented with isolated internal ophthalmoplegia or isolated abducens palsy. The duration from the onset of diplopia to the initial recovery of extraocular movement was $18.8 \pm 14.5$ days ( $n=$ 21 , mean $\pm S D$; range $=7$ to 65 days).

The interval from the beginning of the IVIg treatment to the initial recovery of extraocular movement was $11.7 \pm 12.1$ days ( $n=19$; range $=2$ to 48 days). The most-frequent initially recovered EOM ( $n=22)$ was the LR (17 patients, $77.3 \%)$, followed by the inferior rectus (3 patients, 13.6\%) and medial rectus (2 patients, 9.1\%) muscles. The last-to-recover EOM was the LR in 15 of 18 (83.3\%) patients, while in 1 (5.6\%) case it was the medial rectus muscle and in $2(11.1 \%)$ cases it was the superior rectus muscle.

The additional manifestations of MFS comprised nine (36.0\%) patients with facial nerve palsy and seven (28.0\%) patients with bulbar palsy. No cases involved the optic nerve.

We performed laboratory CSF analyses of 23 patients (since 2 did not consent to the test) at $3.8 \pm 2.1$ days after they presented with their initial symptom. CSF albuminocytologic dissociation was detected in seven (30.4\%) patients. Eighteen of the $24(75.0 \%)$ cases were positive for the IgG anti-GQ1bganglioside antibody as determined by an ELISA.

The age at onset was much higher in the CO group (58.0 \pm 18.4 years, range $=24$ to 75 years) than in the IO group ( $24.9 \pm$ 7.4 years, range $=15$ to 39 years) $(p<0.001)$ (Table 2$)$, as was the proportion of females [7 of 11 patients $(63.6 \%)$ vs. 3 of 14 patients (21.4\%), $p=0.049)$. In addition, CSF albuminocyto-

Table 2. Comparison between $\mathrm{CO}$ and $\mathrm{IO}$

\begin{tabular}{|c|c|c|c|}
\hline & $\operatorname{CO}(n=11)$ & $10(n=14)$ & $p$ \\
\hline Age, years & $58.0 \pm 18.4$ (24 to 75$)$ & $24.9 \pm 7.4$ (15 to 39$)$ & $<0.001^{*}$ \\
\hline Sex, female & 7 (63.6) & $3(21.4)$ & $0.049^{+}$ \\
\hline Antecedent infection & $9(81.8)$ & $13(92.9)$ & $0.565^{\dagger}$ \\
\hline Upper respiratory symptoms & $6(66.7)$ & $6(46.2)$ & N/A \\
\hline Gastrointestinal symptoms & $3(33.3)$ & $7(53.8)$ & N/A \\
\hline Typical MFS with classic triad & $10(90.1)$ & $10(71.4)$ & $0.341^{+}$ \\
\hline CSF albuminocytologic dissociation ${ }^{+}$ & $6 / 10(60.0)$ & $1 / 13(7.7)$ & $0.019^{+}$ \\
\hline CSF protein, $\mathrm{mg} / \mathrm{dL}^{\ddagger}$ & $52.1 \pm 24.3$ & $37.5 \pm 7.0$ & $0.101^{*}$ \\
\hline Mean time from onset to CSF analysis, days ${ }^{\ddagger}$ & $3.3 \pm 1.6$ & $3.9 \pm 2.4$ & $0.648^{*}$ \\
\hline Positivity for lgG anti-G01b antibody & $6 / 11(54.5)$ & $12 / 13(92.3)$ & $0.061^{+}$ \\
\hline Recovery time of ophthalmoplegia from initial symptom onset, weeks ${ }^{5}$ & $22.8 \pm 9.5$ (11 to 37$)$ & $11.4 \pm 6.1$ (5 to 23$)$ & $0.012^{*}$ \\
\hline Facial palsy & $5(45.5)$ & $4(28.6)$ & $0.434^{+}$ \\
\hline Bulbar palsy & $1(9.1)$ & $6(42.9)$ & $0.090^{+}$ \\
\hline Internal ophthalmoplegia & $6(54.5)$ & $4(28.6)$ & $0.241^{+}$ \\
\hline
\end{tabular}

Data are $n(\%)$, mean \pm SD, or mean \pm SD (range) values.

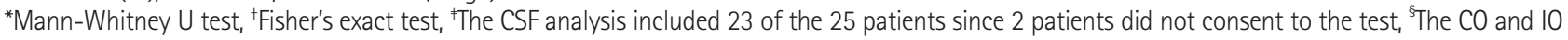
groups included 8 of 11 patients and 12 of 14 patients, respectively.

CO: complete ophthalmoplegia, IgG: immunoglobulin G, IO: incomplete ophthalmoplegia, MFS: Miller Fisher syndrome, N/A: not applicable. 
logic dissociation was more common in the CO group (6 of 10 patients, $60.0 \%$ ) than in the IO group (1 of 13 patients, $7.7 \%)(p=0.019)$. Although not statistically significant, the CSF protein level tended to higher in the CO group (52.1 \pm $24.3 \mathrm{mg} / \mathrm{dL})$ than in the IO group $(37.5 \pm 7.0 \mathrm{mg} / \mathrm{dL})(p=$ 0.101 ), while positivity for the IgG anti-GQ1b-ganglioside antibody tended to higher in the former (12 of 13 patients, $92.3 \%$ ) than the latter ( 6 of 11 patients, $54.5 \%$ ) group ( $p=$ 0.061 ). The recovery time of ophthalmoplegia from the initial symptom onset was faster in the IO group (12 of 14 patients; $11.4 \pm 6.1$ weeks, range $=5$ to 23 weeks) than in the $\mathrm{CO}$ group ( 8 of 11 patients; $22.8 \pm 9.5$ weeks, range $=11$ to 37 weeks) $(p=0.012)$. There was a trend that bulbar palsy was more common in the IO group (6 of 14 patients, $42.9 \%$ ) than in the CO group (1 of 11 patients, 9.1\%), although it was not statistically significant $(p=0.090)$. There were also no significant intergroup differences in the prevalence of facial palsy [5 of 11 patients in the $\mathrm{CO}$ group (45.5\%) and 4 of 14 patients in the IO group (28.6\%), $p=0.434]$ or internal ophthalmoplegia [6 of 11 patients in the $\mathrm{CO}$ group (54.5\%) and 4 of $14 \mathrm{pa}-$ tients in the IO group (28.6\%), $p=0.241$ ).

\section{DISCUSSION}

Ophthalmoplegia is a cardinal sign of various neurologic disorders, but its pattern may differ according to the pathophysiology and location of the lesions. This means that careful evaluation of ophthalmoplegia may provide diagnostic clues regarding the underlying etiology. From this perspective, the findings of the present study could be useful when evaluating ophthalmoplegia in patients with classic or variant forms of MFS. Our results suggest that patients with MFS present with diverse patterns of ocular motility findings. IC or partial ophthalmoplegia was more frequent than $\mathrm{CO}$, and the most frequently involved muscle was the LR, resulting in esotropia.

The traditional approach is for disorders with leading etiologies to be considered in patients presenting with acute ophthalmoplegia, including cerebrovascular disorders, diabetes mellitus, brain tumors, inflammatory disease, trauma, myasthenia gravis (MG), and focal myopathy. MG is a representative disease that should be differentiated from the ophthalmoplegia of MFS. The predominant symptom in MG is asymmetric bilateral or unilateral oculomotor weakness accompanied by ptosis with prominent fatigue. Vertical deviation or exotropia is the most-common form of oculomotor weakness in MG, while total ophthalmoplegia is relatively rare. ${ }^{15}$

The most-prominent oculomotor finding in the present study was LR limitation (100\%). Esotropia was identified in 16 patients, and this was the most-common pattern of ocular deviation (64.0\%). This is similar to limitation of abduction being the most-common finding in previous studies investigating acute ophthalmoplegia without ataxia., ${ }^{2,3}$ The LR was the most-involved and last-to-recover EOM in the present study. Considering the pathophysiology in terms of the LR being the most commonly involved muscle, we suggest that these results are not directly related to only the composition of GQ1b ganglioside. The proportion of GQ1b ganglioside in the abducens nerve is similar to those in the oculomotor and trochlear nerves. ${ }^{16}$ This discrepancy between the anatomical composition of GQ1b ganglioside and neurologic signs is also found in MFS cases with delayed facial nerve involvement. ${ }^{8,17,18}$

Most MFS patients exhibit bilateral and symmetric ophthalmoplegia, ${ }^{1}$ with them rarely exhibiting unilateral ophthalmoplegia. ${ }^{19,20}$ All of the 25 MFS cases in the current study presented with bilateral ophthalmoplegia, but the exact incidence of unilateral ophthalmoplegia in MFS still remains to be investigated thoroughly. A series of patients with acute ophthalmoplegia without ataxia found that unilateral ophthalmoplegia was not uncommon $(23.8-27.3 \%))^{2,3}$ We speculate that the differences in the incidence of unilaterality may result from the severity or extent of disease involvement between the both types; that is, acute ophthalmoplegia without ataxia and MFS. However, this difference needs to be studied in greater depth.

This study compared $\mathrm{CO}$ and IO patients, including performing group comparisons of various clinical findings. We found that the CO group was significantly older and comprised a higher proportion of females than the IO group. In addition, the CO group showed a longer duration to full recovery than the IO group. This result differs from a previous study finding that the type of ophthalmoplegia (CO or IO) did not affect the recovery time. ${ }^{4}$ We cannot explain why the groups showed significant differences in prognosis, other than to speculate that MFS with $\mathrm{CO}$ has a different background from MFS with IO. We think that these findings are due to differences in sex and age. Another interesting finding was the intergroup difference in the incidence of albuminocytologic dissociation, which provides further evidence that these two groups have different immunologic backgrounds.

The present study was subject to some limitations. First, although our hospital is a large referring hospital with a systematized medical recording system, this was a single-center study with a retrospective design, and so selection bias could have been present. Second, we could not evaluate the effects of the type of treatment, because most patients were treated with IVIg (21 patients, 84.0\%). However, to the best of our knowledge our study is the first to focus on the pattern of ophthalmoplegia in MFS patients. The present findings can be used to explain the natural course of oculomotor findings and help physicians understand this disease. 
In conclusion, the LR was the most-involved and last-torecover EOM in MFS patients. Esotropia was the most-common pattern of ocular deviation in the primary position. The CO patients were more likely to be female, were much older, and had a higher incidence rates of facial palsy and albuminocytologic dissociation than the IO patients.

\section{Conflicts of Interest}

The authors have no potential conflicts of interest to disclose.

\section{Acknowledgements}

This study was supported by Dong-A University Hospital and the National Research Foundation of Korea (NRF) grant funded by the Korea government (MSIT) (No. 2016R1A5A2007009).

\section{REFERENCES}

1. Snyder LA, Rismondo V, Miller NR. The Fisher variant of GuillainBarré syndrome (Fisher syndrome). J Neuroophthalmol 2009;29:312324

2. Yuki N, Odaka M, Hirata K. Acute ophthalmoparesis (without ataxia) associated with anti-GQ1b IgG antibody: clinical features. Ophthalmology 2001;108:196-200.

3. Lee SH, Lim GH, Kim JS, Oh SY, Kim JK, Cha JK, et al. Acute ophthalmoplegia (without ataxia) associated with anti-GQ1b antibody. Neurology 2008;71:426-429.

4. Mori M, Kuwabara S, Fukutake T, Yuki N, Hattori T. Clinical features and prognosis of Miller Fisher syndrome. Neurology 2001;56:1104-1106.

5. Chiba A, Kusunoki S, Shimizu T, Kanazawa I. Serum IgG antibody to ganglioside GQ1b is a possible marker of Miller Fisher syndrome. Ann Neurol 1992;31:677-679.

6. Mizoguchi K. Anti-GQ1b IgG antibody activities related to the severity of Miller Fisher syndrome. Neurol Res 1998;20:617-624.

7. Kim JK, Kim BJ, Shin HY, Shin KJ, Nam TS, Oh J, et al. Acute bulbar palsy as a variant of Guillain-Barré syndrome. Neurology 2016;86:742-
747.

8. Kim JK, Kim BJ, Shin HY, Shin KJ, Nam TS, Seok JI, et al. Does delayed facial involvement implicate a pattern of "descending reversible paralysis" in Fisher syndrome? Clin Neurol Neurosurg 2015;135:1-5.

9. Chan JW. Optic neuritis in anti-GQ1b positive recurrent Miller Fisher syndrome. Br J Ophthalmol 2003;87:1185-1186.

10. Ryu WY, Kim JK, Jin SW, Shim DH. Acute angle-closure glaucoma in a patient with Miller Fisher syndrome without pupillary dysfunction. J Glaucoma 2015;24:e5-e6.

11. Wakerley BR, Uncini A, Yuki N; GBS Classification Group; GBS Classification Group. Guillain-Barré and Miller Fisher syndromes--new diagnostic classification. Nat Rev Neurol 2014;10:537-544.

12. Keane JR. Bilateral ocular paralysis: analysis of 31 inpatients. Arch Neurol 2007;64:178-180.

13. Thurtell MJ, Halmagyi GM. Complete ophthalmoplegia: an unusual sign of bilateral paramedian midbrain-thalamic infarction. Stroke 2008; 39:1355-1357.

14. Kim JK, Bae JS, Kim DS, Kusunoki S, Kim JE, Kim JS, et al. Prevalence of anti-ganglioside antibodies and their clinical correlates with Guillain-Barré syndrome in Korea: a nationwide multicenter study. $J$ Clin Neurol 2014;10:94-100.

15. Ahn J, Park KS, Kim JS, Hwang JM. Efficacy of gaze photographs in diagnosing ocular myasthenia gravis. J Clin Neurol 2018;14:333-338.

16. Chiba A, Kusunoki S, Obata H, Machinami R, Kanazawa I. Ganglioside composition of the human cranial nerves, with special reference to pathophysiology of Miller Fisher syndrome. Brain Res 1997;745:32-36.

17. Tan CY, Yuki N, Shahrizaila N. Delayed facial palsy in Miller Fisher syndrome. J Neurol Sci 2015;358:409-412.

18. Tatsumoto M, Misawa S, Kokubun N, Sekiguchi Y, Hirata K, Kuwabara S, et al. Delayed facial weakness in Guillain-Barré and Miller Fisher syndromes. Muscle Nerve 2015;51:811-814.

19. Smith J, Clarke L, Severn P, Boyce R. Unilateral external ophthalmoplegia in Miller Fisher syndrome: case report. BMC Ophthalmol 2007; 7:7.

20. Susuki K, Yuki N, Muramatsu M, Hirata K. Unilateral ophthalmoparesis and limb ataxia associated with anti-GQ1b IgG antibody. J Neurol 2000;247:652-653. 